\title{
ON THE DENSITY AND MOMENTS OF THE TIME OF RUIN WITH EXPONENTIAL CLAIMS
}

\author{
BY
}

Steve Drekic and Gordon E. Willmot

\begin{abstract}
The probability density function of the time of ruin in the classical model with exponential claim sizes is obtained directly by inversion of the associated Laplace transform. This result is then used to obtain explicit closed-form expressions for the moments. The form of the density is examined for various parameter choices.
\end{abstract}

\section{KEYWORDS}

Laplace transform, Bessel function, Gauss hypergeometric series, Gerber-Shiu equation, compound geometric tail, ruin probability.

\section{INTRODUCTION AND PRELIMINARIES}

Consider the classical risk process, whereby the number of claims process $\left\{N_{t}\right.$; $t \geq 0\}$ is a Poisson process with rate $\lambda$. The individual claims $\left\{Y_{1}, Y_{2}, \ldots\right\}$ are an independent and identically distributed (iid) sequence of positive random variables with distribution function (df) $H(y)=1-\bar{H}(y)=\operatorname{Pr}(Y \leq y)$, where $Y$ is an arbitrary $Y_{n}$. Premiums are paid continuously at rate $c$ per unit time, where $c=$ $\lambda E\{Y\}(1+\theta)$ with $\theta>0$ the relative security loading. The surplus process $\left\{U_{t}\right.$; $t \geq 0\}$, beginning with initial surplus $x$, is defined by $U_{t}=x+c t-\sum_{n=1}^{N_{t}} Y_{n}$.

The time to ruin is $T=\inf \left\{t: U_{t}<0\right\}$ with $T=\infty$ if $U_{t} \geq 0$ for all $t \geq 0$. The Laplace transform of the (defective) distribution of the time to ruin satisfies the so-called Gerber-Shiu defective renewal equation, and is well known to be the tail of a compound geometric distribution (e.g. Willmot and Lin, 2001, Section 9.2). That is,

$$
\tilde{f}(\delta)=E\left\{e^{-\delta T} 1(T<\infty)\right\}=\sum_{n=1}^{\infty}(1-\phi) \phi^{n} \bar{F}^{*} n(x),
$$

where 


$$
\phi=\frac{\int_{0}^{\infty} e^{-\rho y} \bar{H}(y) d y}{(1+\theta) E\{Y\}},
$$

with $\rho=\rho(\delta)$ the unique non-negative root of Lundberg's equation

$$
\lambda \int_{0}^{\infty} e^{-\rho y} d H(y)=\lambda+\delta-c p
$$

and $\bar{F}^{* n}(x)$ is the tail of the $n$-fold convolution of the $\mathrm{df} F(x)=1-\bar{F}(x)$ satisfying

$$
\bar{F}(x)=\frac{\int_{0}^{\infty} e^{-\rho y} \bar{H}(x+y) d y}{\int_{0}^{\infty} e^{-\rho y} \bar{H}(y) d y} .
$$

In (1.1) above, $1(\mathcal{A})$ denotes the indicator function of the event $\mathcal{A}$, which is equal to 1 if the event occurs, and is 0 otherwise. Of course, when $\delta=0$, one recovers the ruin probability $\psi(x)=\operatorname{Pr}(T<\infty)=E\{1(T<\infty)\}=\tilde{f}(0)$.

In the special case with exponential claim amounts, i.e. $\bar{H}(y)=e^{-\mu y}, y \geq 0$, then $\bar{F}(x)=\bar{H}(x)$ from (1.4), and (1.1) simplifies to (e.g. Willmot and Lin, 2001, equation 4.1.12)

$$
\tilde{f}(\delta)=\phi e^{-\mu(1-\phi) x},
$$

where $\phi=\mu /\{(\mu+\rho)(1+\theta)\}$ from (1.2), and (1.3) becomes $c \rho^{2}-(\lambda+\delta-c \mu) \rho-$ $\delta \mu=0$, in turn implying that

$$
\rho=\frac{\lambda+\delta-c \mu+\sqrt{(\lambda+\delta-c \mu)^{2}+4 \delta c \mu}}{2 c}
$$

or equivalently

$$
\rho=\frac{\lambda+\delta-c \mu+\sqrt{(\lambda+\delta+c \mu)^{2}-4 \lambda c \mu}}{2 c} .
$$

This result is also given by Asmussen (2000, p. 99).

In the next section, we invert the Laplace transform (1.5) directly to get the probability density function (pdf) $f(t)$ satisfying $\tilde{f}(\delta)=\int_{0}^{\infty} e^{-\delta t} f(t) d t$. The pdf $g(t)$ of the time of ruin $T_{c}=T \mid T<\infty$ (conditional on ruin occurring) is then obtained. The pdf is in a simpler form than that which results from the relationship between the finite time ruin probabilities of the present model and the transient virtual waiting time in the M/M/1 queue (e.g. Seal, 1978, Chapter 2, or Asmussen, 2000, p. 101). We remark that an alternative derivation of this pdf is possible using the proof of Corollary 2 of Wang and Liu (2002). 
In Section 3, we use this pdf to derive explicit expressions for the moments which do not involve recursive calculations of intermediate quantities, as is the case for the formulas in Lin and Willmot (2000). Moreover, only elementary functions are involved. Finally, the form of the pdf is examined for various parameter choices in Section 4.

\section{DeRIVATION OF THE DENSITY FUNCTION}

In order to invert the Laplace transform (1.5), first note that with $\bar{H}(y)=e^{-\mu y}$, (1.2) and (1.3) imply that

$$
\phi=\frac{\int_{0}^{\infty} e^{-\rho y} d H(y)}{1+\theta}=\frac{\lambda+\delta-c \rho}{\lambda(1+\theta)} .
$$

Therefore, since $c \mu=\lambda(1+\theta)$ in this case, (1.6) results in

$$
\begin{aligned}
\phi & =\frac{1}{\lambda(1+\theta)}\left\{\lambda+\delta-\frac{\lambda+\delta-c \mu+\sqrt{(\lambda+\delta+c \mu)^{2}-4 \lambda c \mu}}{2}\right\} \\
& =\frac{\lambda+\delta+c \mu-\sqrt{(\lambda+\delta+c \mu)^{2}-4 \lambda c \mu}}{2 c \mu}
\end{aligned}
$$

and so $\phi$ now has an explicit representation in terms of $\delta$. Before inserting (2.1) into (1.5), note that (1.5) may be expanded as

$$
\tilde{f}(\delta)=e^{-\mu x} \sum_{n=0}^{\infty} \phi^{n+1} \frac{(\mu x)^{n}}{n !} .
$$

Substitution of (2.1) into (2.2) yields

$$
\begin{aligned}
\tilde{f}(\delta) & =e^{-\mu x} \sum_{n=0}^{\infty} \frac{(\mu x)^{n}}{n !}\left\{\frac{\lambda+\delta+c \mu-\sqrt{(\lambda+\delta+c \mu)^{2}-4 \lambda c \mu}}{2 c \mu}\right\}^{n+1} \\
& =\frac{e^{-\mu x}}{2 c \mu} \sum_{n=0}^{\infty} \frac{\left(\frac{x}{2 c}\right)^{n}}{n !}\left\{s-\sqrt{s^{2}-a^{2}}\right\}^{n+1}
\end{aligned}
$$

where $s=\lambda+\delta+c \mu$ and $a=2 \sqrt{\lambda c \mu}$. If we let $f(t)=L^{-1}[\tilde{f}(\delta)]$ represent the inverse Laplace transform of $\tilde{f}(\delta)$, then inverting both sides of $(2.3)$ results in

$$
f(t)=\frac{e^{-\mu x}}{2 c \mu} \sum_{n=0}^{\infty} \frac{\left(\frac{x}{2 c}\right)^{n}}{n !} L^{-1}\left[\left\{s-\sqrt{s^{2}-a^{2}}\right\}^{n+1}\right] .
$$


If we now make use of transform results in Abramowitz and Stegun (1972, p. 1021, formula 29.2.12) and Schiff (1999, p. 214, $2^{\text {nd }}$ transform pair), then

$$
L^{-1}\left[\left\{s-\sqrt{s^{2}-a^{2}}\right\}^{n+1}\right]=e^{-(\lambda+c \mu) t} \frac{(n+1)(2 \sqrt{\lambda c \mu})^{n+1} I_{n+1}(2 t \sqrt{\lambda c \mu})}{t}
$$

where

$$
I_{v}(y)=\sum_{k=0}^{\infty} \frac{\left(\frac{y}{2}\right)^{2 k+v}}{k !(k+v) !}
$$

is the modified Bessel function of the first kind of order $v$ (e.g. Abramowitz and Stegun, 1972, p. 375, formula 9.6.10, or Schiff, 1999, p. 102). Substituting (2.5) into (2.4) then yields, again using $c=\lambda(1+\theta) / \mu$,

$$
f(t)=\frac{e^{-\mu x} e^{-\lambda(2+\theta) t}}{t \sqrt{1+\theta}} \sum_{n=0}^{\infty} \frac{(n+1)\left(\frac{\mu x}{\sqrt{1+\theta}}\right)^{n} I_{n+1}(2 \lambda t \sqrt{1+\theta})}{n !}, t>0 .
$$

Since $T_{c}$ has pdf $g(t)=f(t) / \psi(x)$ where (e.g. Asmussen, 2000, p. 63) $\psi(x)=e^{-R x} /$ $(1+\theta)$ with $R=\mu \theta /(1+\theta)$, we obtain from (2.7) that

$$
g(t)=\frac{\sqrt{1+\theta} e^{\frac{-\mu x}{1+\theta}} e^{-\lambda(2+\theta) t}}{t} \sum_{n=0}^{\infty} \frac{(n+1)\left(\frac{\mu x}{\sqrt{1+\theta}}\right)^{n} I_{n+1}(2 \lambda t \sqrt{1+\theta})}{n !}, t>0 .
$$

\section{Derivation OF THE MOMENTS}

We now consider the moments $m_{k}=E\left\{T^{k} 1(T<\infty)\right\}, k=1,2, \ldots$. To obtain $m_{k}$, we use (2.7) to get

$$
\begin{aligned}
m_{k} & =\int_{0}^{\infty} t^{k} f(t) d t \\
& =\frac{e^{-\mu x}}{\sqrt{1+\theta}} \sum_{n=0}^{\infty} \frac{(n+1)\left(\frac{\mu x}{\sqrt{1+\theta}}\right)^{n}}{n !} \int_{0}^{\infty} t^{k-1} e^{-\lambda(2+\theta) t} I_{n+1}(2 \lambda t \sqrt{1+\theta}) d t .
\end{aligned}
$$

From Gradshteyn and Ryzhik (1994, p. 732), we have that

$$
\int_{0}^{\infty} e^{-\alpha t} J_{v}(\beta t) t^{\sigma-1} d t=\frac{\left(\frac{\beta}{2}\right)^{v} \Gamma(v+\sigma)}{\sqrt{\left(\alpha^{2}+\beta^{2}\right)^{v+\sigma}} \Gamma(v+1)} \mathcal{F}\left(\frac{v+\sigma}{2}, \frac{1-\sigma+v}{2} ; v+1 ; \frac{\beta^{2}}{\alpha^{2}+\beta^{2}}\right)
$$

for $\operatorname{Re}(v+\sigma)>0, \operatorname{Re}(\alpha+i \beta)>0$, and $\operatorname{Re}(\alpha-i \beta)>0$. In (3.1) above, $\mathcal{F}(a, b ; c ; z)$ represents the Gauss hypergeometric series (see Abramowitz and Stegun, 1972, 
p. 556) and $J_{v}(i z)=i^{v} I_{v}(z)$ for integral $v$ (see Gradshteyn and Ryzhik, 1994, p. 961) where $i$ satisfies $i^{2}=-1$. Defining $\alpha=\lambda(2+\theta), v=n+1, \beta=2 \lambda i \sqrt{1+\theta}$, and $\sigma=k$, it is easily verified that all of the above conditions hold true in order for (3.1) to apply. Therefore,

$$
\begin{aligned}
\int_{0}^{\infty} e^{-\alpha t} J_{v}(\beta t) t^{\sigma-1} d t & =\frac{i^{n+1}(\lambda \sqrt{1+\theta})^{n+1}(n+k) !}{(n+1) ! \sqrt{\left\{\lambda^{2}(2+\theta)^{2}-4 \lambda^{2}(1+\theta)\right\}^{n+k+1}}} \\
& \times \mathcal{F}\left(\frac{n+k+1}{2}, \frac{n-k+2}{2} ; n+2 ; \frac{-4 \lambda^{2}(1+\theta)}{\lambda^{2}(2+\theta)^{2}-4 \lambda^{2}(1+\theta)}\right) \\
& =\frac{i^{n+1} \sqrt{1+\theta}}{\lambda^{k} \theta^{k+1}} \cdot\left(\frac{\sqrt{1+\theta}}{\theta}\right)^{n} \cdot \frac{(n+k) !}{(n+1) !} \\
& \times \mathcal{F}\left(\frac{n+k+1}{2}, \frac{n-k+2}{2} ; n+2 ; \frac{-4(1+\theta)}{\theta^{2}}\right) .
\end{aligned}
$$

Since $J_{v}(\beta t)=J_{n+1}(2 \lambda i t \sqrt{1+\theta})=i^{n+1} I_{n+1}(2 \lambda t \sqrt{1+\theta})$, it follows that

$$
\begin{aligned}
\int_{0}^{\infty} t^{k-1} e^{-\lambda(2+\theta) t} I_{n+1}(2 \lambda t \sqrt{1+\theta}) d t & =\frac{\sqrt{1+\theta}}{\lambda^{k} \theta^{k+1}} \cdot\left(\frac{\sqrt{1+\theta}}{\theta}\right)^{n} \cdot \frac{(n+k) !}{(n+1) !} \\
& \times \mathcal{F}\left(\frac{n+k+1}{2}, \frac{n-k+2}{2} ; n+2 ; \frac{-4(1+\theta)}{\theta^{2}}\right),
\end{aligned}
$$

and so

$$
m_{k}=\frac{e^{-\mu x}}{\lambda^{k} \theta^{k+1}} \sum_{n=0}^{\infty} \frac{(n+k) !\left(\frac{\mu x}{\theta}\right)^{n}}{(n !)^{2}} \mathcal{F}\left(\frac{n+k+1}{2}, \frac{n-k+2}{2} ; n+2 ; \frac{-4(1+\theta)}{\theta^{2}}\right) .
$$

We now consider the simplification of the Gauss hypergeometric series in (3.2). For this purpose, define $a=(n+k+1) / 2, b=(n-k+2) / 2$, and $z=-4(1+\theta) / \theta^{2}$. Note that $a-b+1 / 2=k$ and $a+b+1 / 2=n+2$. Therefore, applying formula 15.3.23 of Abramowitz and Stegun (1972, p. 560), we obtain

$$
\begin{aligned}
& \mathcal{F}\left(\frac{n+k+1}{2}, \frac{n-k+2}{2} ; n+2 ; \frac{-4(1+\theta)}{\theta^{2}}\right) \\
& =\left(\frac{1}{2}+\frac{1}{2} \sqrt{1+\frac{4(1+\theta)}{\theta^{2}}}\right)^{-2\left(\frac{n+k+1}{2}\right)} \mathcal{F}\left(n+k+1, k ; n+2 ; \frac{\sqrt{1+\frac{4(1+\theta)}{\theta^{2}}}-1}{\sqrt{1+\frac{4(1+\theta)}{\theta^{2}}}+1}\right) \\
& =\left(\frac{1}{2}+\frac{1}{2} \cdot \frac{\theta+2}{\theta}\right)^{-(n+k+1)} \mathcal{F}\left(n+k+1, k ; n+2 ; \frac{\frac{\theta+2}{\theta}-1}{\frac{\theta+2}{\theta}+1}\right) \\
& =\left(\frac{\theta}{1+\theta}\right)^{k+1}\left(\frac{\theta}{1+\theta}\right)^{n} \mathcal{F}\left(n+k+1, k ; n+2 ; \frac{1}{1+\theta}\right) .
\end{aligned}
$$


Substituting (3.3) into (3.2) then yields

$$
m_{k}=\frac{\omega^{k+1} e^{-\mu x}}{\lambda^{k}} \sum_{n=0}^{\infty} \frac{(n+k) !(\mu x \omega)^{n}}{(n !)^{2}} \mathcal{F}(n+k+1, k ; n+2 ; \omega)
$$

where we define $\omega=(1+\theta)^{-1}$. From formulas 6.1.22 and 15.2.3 of Abramowitz and Stegun (1972, pp. 256, 557), it follows that

$$
\mathcal{F}\left(a^{\prime}+m, b^{\prime} ; c ; \omega\right)=\frac{\Gamma\left(a^{\prime}\right)}{\Gamma\left(a^{\prime}+m\right)} \omega^{-\left(a^{\prime}-1\right)} \frac{d^{m}}{d \omega^{m}}\left[\omega^{a^{\prime}+m-1} \mathcal{F}\left(a^{\prime}, b^{\prime} ; c ; \omega\right)\right] .
$$

In (3.5) and in what follows, we adopt the notational convention that the $0^{\text {th }}$ derivative of a function is the function itself. If we now choose $a^{\prime}=n+2, b^{\prime}=k$, $c=n+2$, and $m=k-1$, then (3.5) implies that

$$
\begin{aligned}
\mathcal{F}(n+k+1, k ; n+2 ; \omega) & =\frac{\Gamma(n+2)}{\Gamma(n+k+1)} \omega^{-(n+1)} \frac{d^{k-1}}{d \omega^{k-1}}\left[\omega^{n+k} \mathcal{F}(n+2, k ; n+2 ; \omega)\right] \\
& =\frac{(n+1) !}{(n+k) !} \omega^{-(n+1)} \frac{d^{k-1}}{d \omega^{k-1}}\left[\omega^{n+k} \mathcal{F}(n+2, k ; n+2 ; \omega)\right] .
\end{aligned}
$$

Furthermore, from formulas 15.1.1 and 15.1.8 of Abramowitz and Stegun (1972, p. 556), it follows that

$$
\mathcal{F}(n+2, k ; n+2 ; \omega)=\mathcal{F}(k, n+2 ; n+2 ; \omega)=(1-\omega)^{-k},
$$

and substitution into (3.6) yields

$$
\mathcal{F}(n+k+1, k ; n+2 ; \omega)=\frac{(n+1) !}{(n+k) !} \omega^{-(n+1)} \frac{d^{k-1}}{d \omega^{k-1}}\left[\omega^{n+k}(1-\omega)^{-k}\right] .
$$

Therefore, (3.4) becomes

$$
\begin{aligned}
m_{k} & =\left.\frac{e^{-\mu x}}{\lambda^{k}(1+\theta)^{k}} \sum_{n=0}^{\infty} \frac{(n+1)(\mu x)^{n}}{n !} \frac{d^{k-1}}{d \omega^{k-1}}\left[\omega^{n+k}(1-\omega)^{-k}\right]\right|_{\omega=1 /(1+\theta)} \\
& =\left.\frac{e^{-\mu x}}{\lambda^{k}(1+\theta)^{k}} \cdot \frac{d^{k-1}}{d \omega^{k-1}}\left[\omega^{k}(1-\omega)^{-k} \sum_{n=0}^{\infty} \frac{(n+1)(\mu x \omega)^{n}}{n !}\right]\right|_{\omega=1 /(1+\theta)} .
\end{aligned}
$$

However, note that

$$
\sum_{n=0}^{\infty} \frac{(n+1)(\mu \times \omega)^{n}}{n !}=\sum_{n=1}^{\infty} \frac{(\mu \times \omega)^{n}}{(n-1) !}+\sum_{n=0}^{\infty} \frac{(\mu \times \omega)^{n}}{n !}=e^{\mu \times \omega}(1+\mu \times \omega) .
$$

Thus, 


$$
m_{k}=\left.\frac{e^{-\mu x}}{\lambda^{k}(1+\theta)^{k}} \cdot \frac{d^{k-1}}{d \omega^{k-1}}\left[\omega^{k}(1-\omega)^{-k} e^{\mu x \omega}(1+\mu x \omega)\right]\right|_{\omega=1 /(1+\theta)} .
$$

It is clear from (3.7) that the $k^{\text {th }}$ moment $m_{k}$ involves only elementary functions. In order to evaluate the derivative in (3.7), we define $p(\omega)=\omega^{k}(1-\omega)^{-k}$ and $q(\omega)=e^{\mu x \omega}(1+\mu x \omega)$. We apply Leibniz's product rule theorem (e.g. Abramowitz and Stegun, 1972, p. 12) to obtain

$$
m_{k}=\frac{e^{-\mu x}}{\lambda^{k}(1+\theta)^{k}} \sum_{j=0}^{k-1}\left(\begin{array}{c}
k-1 \\
j
\end{array}\right) p^{(j)}\left(\frac{1}{1+\theta}\right) q^{(k-1-j)}\left(\frac{1}{1+\theta}\right),
$$

where $p^{(n)}(\omega)$ and $q^{(n)}(\omega)$ denote the $n^{\text {th }}$ derivatives of $p$ and $q$, respectively, evaluated at $\omega$. It is not difficult to prove via induction that

$$
q^{(n)}(\omega)=(\mu x)^{n} e^{\mu x \omega}(n+1+\mu x \omega) .
$$

Therefore,

$$
\begin{aligned}
e^{-\mu x} q^{(k-1-j)}\left(\frac{1}{1+\theta}\right) & =e^{-\mu x\left(1-\frac{1}{1+\theta}\right)}(\mu x)^{k-1-j}\left(k-j+\frac{\mu x}{1+\theta}\right) \\
& =\psi(x)(1+\theta)(\mu x)^{k-1-j}\left(k-j+\frac{\lambda x}{c}\right) .
\end{aligned}
$$

Thus, substitution into (3.8) implies that

$$
m_{k}=\frac{\psi(x)}{\lambda^{k}(1+\theta)^{k-1}} \sum_{j=0}^{k-1}\left(\begin{array}{c}
k-1 \\
j
\end{array}\right)(\mu x)^{k-1-j}\left(k-j+\frac{\lambda x}{c}\right) p^{(j)}\left(\frac{1}{1+\theta}\right) .
$$

To evaluate the remaining derivative in (3.10), we again apply Leibniz's product rule theorem to obtain

$$
\begin{aligned}
p^{(j)}(\omega) & =\sum_{n=0}^{j}\left(\begin{array}{l}
j \\
n
\end{array}\right) \frac{k !}{(k+n-j) !} \omega^{k-j+n} \frac{(k+n-1) !}{(k-1) !}(1-\omega)^{-k-n} \\
& =j ! \sum_{n=0}^{j}\left(\begin{array}{c}
k \\
j-n
\end{array}\right)\left(\begin{array}{c}
k+n-1 \\
n
\end{array}\right) \omega^{k-j+n}(1-\omega)^{-k-n} .
\end{aligned}
$$

Hence,

$$
p^{(j)}\left(\frac{1}{1+\theta}\right)=j !(1+\theta)^{j} \sum_{n=0}^{j}\left(\begin{array}{c}
k \\
j-n
\end{array}\right)\left(\begin{array}{c}
k+n-1 \\
n
\end{array}\right) \theta^{-k-n} .
$$


Again, substitution into (3.10) yields

$$
\begin{aligned}
m_{k} & =\frac{\psi(x)}{\lambda^{k}} \sum_{j=0}^{k-1} \frac{(k-1) !}{(k-1-j) !}\left(\frac{\mu x}{1+\theta}\right)^{k-1-j}\left(k-j+\frac{\lambda x}{c}\right) \\
& \times \sum_{n=0}^{j}\left(\begin{array}{c}
k \\
j-n
\end{array}\right)\left(\begin{array}{c}
k+n-1 \\
n
\end{array}\right) \theta^{-k-n} .
\end{aligned}
$$

To summarize,

$m_{k}=\psi(x) \frac{(k-1) !}{\lambda^{k}} \sum_{j=0}^{k-1} \frac{\left(\frac{\lambda x}{c}\right)^{k-1-j}}{(k-1-j) !}\left(k-j+\frac{\lambda x}{c}\right) \sum_{n=0}^{j}\left(\begin{array}{c}k \\ j-n\end{array}\right)\left(\begin{array}{c}k+n-1 \\ n\end{array}\right) \theta^{-k-n}$.

Finally, dividing (3.11) by $\psi(x)$ immediately yields the following explicit expression for the $k^{\text {th }}$ moment of $T_{c}, k=1,2, \ldots$ :

$$
E\left\{T_{c}^{k}\right\}=\frac{(k-1) !}{\lambda^{k}} \sum_{j=0}^{k-1} \frac{\left(\frac{\lambda x}{c}\right)^{k-1-j}}{(k-1-j) !}\left(k-j+\frac{\lambda x}{c}\right) \sum_{n=0}^{j}\left(\begin{array}{c}
k \\
j-n
\end{array}\right)\left(\begin{array}{c}
k+n-1 \\
n
\end{array}\right) \theta^{-k-n}
$$

We note that (3.12) is an improvement over the recursive procedure developed by Lin and Willmot (2000, Example 6.1, pp. 41-2) for moments of $T_{c}$. In fact, through the use of (3.12), the following moment-based quantities, which are routinely used in statistical applications to describe the location, spread, skewness, and heavy-tailedness of a probability distribution (e.g. Stuart and Ord, 1994), are easily obtained for $T_{c}$ (recalling that the adjustment coefficient introduced in Section 2 is $R=\mu \theta /(1+\theta))$ :

Mean

$$
\frac{(1+\theta)(R x+\theta)}{c \theta^{2} \mu}
$$

Variance

$$
\frac{(1+\theta)^{2}[2 R x(1+\theta)+\theta(2+\theta)]}{c^{2} \theta^{4} \mu^{2}},
$$

Coefficient of Variation (i.e. $E\left\{\left[T_{c}-E\left\{T_{c}\right\}\right]^{2}\right\}^{1 / 2} / E\left\{T_{c}\right\}$ )

$$
\frac{\sqrt{2 R x(1+\theta)+\theta(2+\theta)}}{R x+\theta}
$$

Coefficient of Skewness (i.e. $E\left\{\left[T_{c}-E\left\{T_{c}\right\}\right]^{3}\right\} / E\left\{\left[T_{c}-E\left\{T_{c}\right\}\right]^{2}\right\}^{3 / 2}$ )

$$
\frac{2[3 R x(1+\theta)(2+\theta)+\theta\{6+\theta(6+\theta)\}]}{\sqrt{2 R x(1+\theta)+\theta(2+\theta)}^{3}},
$$


Coefficient of Kurtosis (i.e. $E\left\{\left[T_{c}-E\left\{T_{c}\right\}\right]^{4}\right\} / E\left\{\left[T_{c}-E\left\{T_{c}\right\}\right]^{2}\right\}^{2}$ )

$$
\frac{3\left[4 R^{2} x^{2}(1+\theta)^{2}+4 R x(1+\theta)\{10+3 \theta(4+\theta)\}+\theta(2+\theta)\{20+\theta(22+3 \theta)\}\right]}{[2 R x(1+\theta)+\theta(2+\theta)]^{2}} .
$$

The formulas for the mean and variance agree with those obtained by Lin and Willmot (2000, pp. 41-2). However, Lin and Willmot only derive explicit expressions for $m_{1}$ and $m_{2}$ whereas (3.11) explicitly provides the general form for any $k$.

\section{NumericAl EXAMPLes}

In this section, we present several plots of the pdf $g(t)$ given by (2.8). All examples were carried out with $\mu=1$, so that we might examine the impact of the
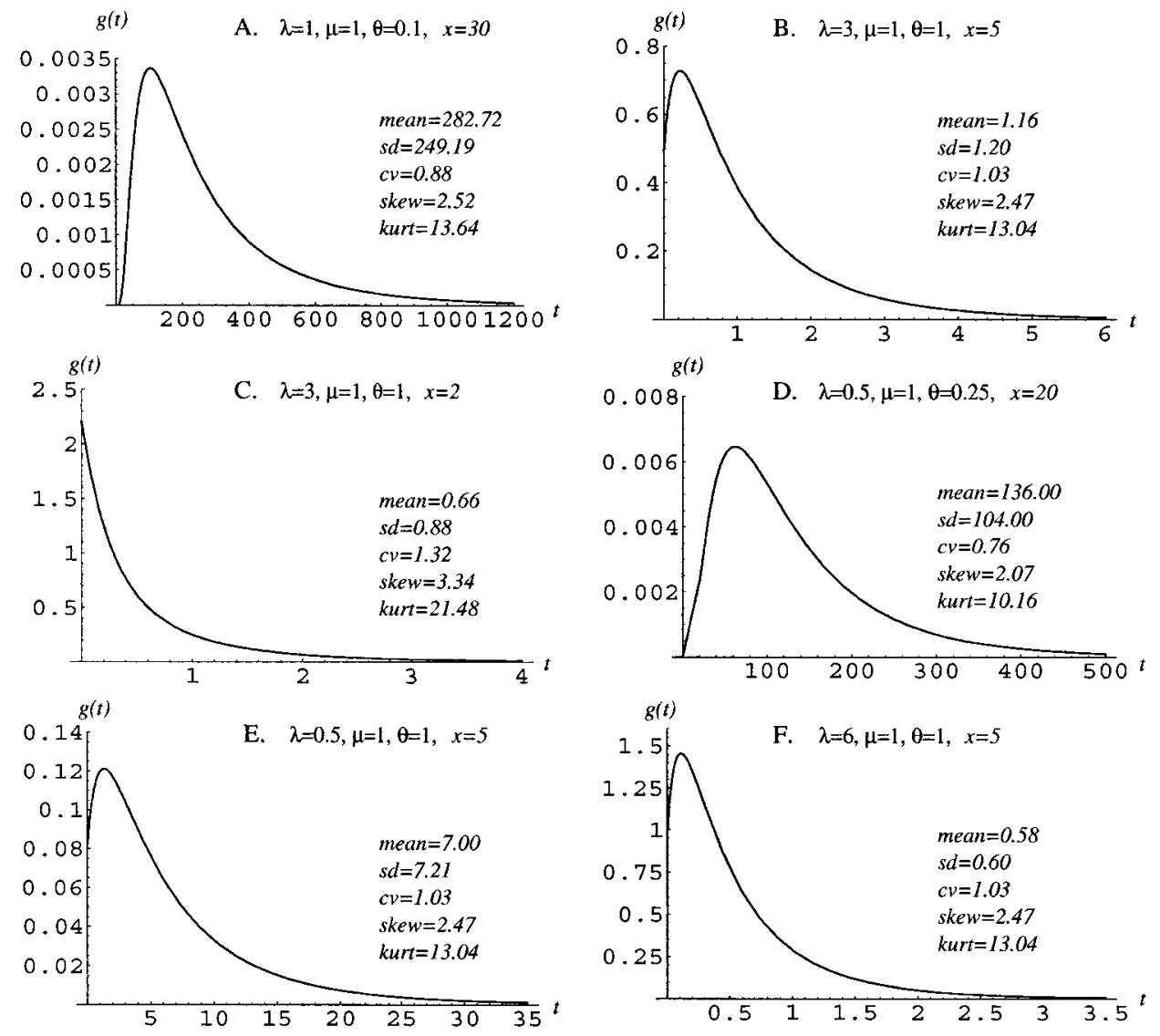

Figure 1: Several plots of the density function $g(t)$ for $T_{c}$. 
remaining parameters (namely, $\lambda, \theta$, and $x$ ) on the distribution of $T_{c}$. Plots of $g(t)$ in Figure 1 were generated using the computational package Mathematica. In particular, Mathematica possesses the built-in function BesselI (Wolfram, 1999 , p. 767) which enables the numerical evaluation of the modified Bessel function given by (2.6). Corresponding to each pdf plot of $T_{c}$, we have calculated (to 2 decimal places of accuracy) its mean, standard deviation (denoted " $s d$ " in Figure 1), coefficient of variation (denoted " $c v$ " in Figure 1), coefficient of skewness (denoted "skew" in Figure 1), and coefficient of kurtosis (denoted "kurt" in Figure 1).

We remark from the plots that the time of ruin $T_{c}$ does not exhibit consistent monotonic behaviour in terms of its reliability classification. In particular, plots $\mathrm{A}$ and $\mathrm{D}$ have $c v<1$ which is inconsistent with decreasing failure rate behaviour whereas plots $\mathrm{B}, \mathrm{C}, \mathrm{E}$, and $\mathrm{F}$ are inconsistent with increasing failure rate behaviour since $c v>1$ (e.g. Willmot and Lin, 2001, p. 26). Moreover, for parameter values $\lambda=3, \theta=4$, and $x=5$, it is curious to note that a plot of $g(t)$ closely resembles the pdf of an exponential distribution with mean $1 / 6$, although it is not reproduced here.

Finally, we note that plots B, E, and F all have the exact same values for $c v$, skew, and kurt. As we look more closely at the parameter settings in these three examples, we observe that it is only $\lambda$ which varies while $\theta$ and $x$ both remain unchanged. The fact that these moment-based quantities do not change is ultimately revealed upon analysis of formulas (3.15), (3.16), and (3.17) which do not involve $\lambda$. In fact, it is straightforward to show that this behaviour holds more generally for any choice of claim amount distribution possessing a sufficient number of moments.

\section{ACKNOWLEDGEMENTS}

This research was supported by the Natural Sciences and Engineering Research Council of Canada. Support for the second author from the Munich Reinsurance Company is also gratefully acknowledged.

\section{REFERENCES}

Abramowitz, M. and Stegun, I. (1972) Handbook of Mathematical Functions: With Formulas, Graphs, and Mathematical Tables. National Bureau of Standards, Washington.

Asmussen, S. (2000) Ruin Probabilities. World Scientific, Singapore.

Gradshteyn, I. and Ryzhik, I. (1994) Table of Integrals, Series, and Products (Fifth Edition). Academic Press, San Diego.

Lin, X. and Willmot, G. (2000) "The moments of the time of ruin, the surplus before ruin, and the deficit at ruin", Insurance: Mathematics and Economics, 27, 19-44.

SCHIFF, J. (1999) The Laplace Transform: Theory and Applications. Springer-Verlag, New York. Seal, H. (1978) Survival Probabilities. John Wiley \& Sons, New York.

StuART, A. and Ord, J. (1994) Kendall's Advanced Theory of Statistics, Volume 1: Distribution Theory. John Wiley \& Sons, New York.

WANG, R. and LIU, H. (2002) "On the ruin probability under a class of risk processes", ASTIN Bulletin, 32, 81-90. 
Willmot, G. and Lin, X. (2001) Lundberg Approximations for Compound Distributions with Insurance Applications. Springer-Verlag, New York.

Wolfram, S. (1999) The Mathematica Book (Fourth Edition). Cambridge University Press, New York.

\author{
Steve Drekic and Gordon E. Willmot \\ Department of Statistics and Actuarial Science \\ University of Waterloo \\ Waterloo, Ontario N2L $3 G 1$ \\ Canada \\ E-mail:sdrekic@math.uwaterloo.ca \\ gewillmo@icarus.uwaterloo.ca
}

